\title{
Analyse des Déterminants de la Résilience aux Changements Climatiques des Cultivateurs du Département de Fresco, Côte d'Ivoire
}

\author{
N'drin Owo Jean Arnaud, \\ Doctorant à l'UFR Anthropologie, \\ Université Félix Houphouët Boigny Cocody \\ Konan-Waidhet Arthur Brice, \\ Maître de Conférences, Télédétection et SIG, \\ UFR Environnement, Université Jean Lorognon Guédé \\ Kienon-Kabore Timpoko Hélène, \\ Professeure Titulaire en Histoire de l'Archéologie, Anthropologie, \\ Université Félix Houphouët Boigny Cocody
}

Doi: 10.19044/esj.2019.v15n18p288 URL:http://dx.doi.org/10.19044/esj.2019.v15n18p288

\section{Résumé}

Les changements climatiques sont un défi mondial auquel toute l'humanité entière est confrontée. Les cultivateurs, en plus des nombreux problèmes auxquels ils sont déjà confrontés, doivent désormais faire face à ce nouveau phénomène. L'adaptation semble être actuellement l'une des meilleures alternatives. L'analyse de cette adaptation appelée résilience permet d'en connaitre les facteurs explicatifs. C'est dans cette perspective que la présente étude s'inscrit, avec pour objectif d'analyser la résilience aux changements climatiques des cultivateurs dans le département de Fresco. Sur la base de la théorie des dynamiques adaptatives de Bennett, l'hypothèse de travail est que les cultivateurs résilients du département de Fresco adoptent des cultures à cours cycle et associent les cultures pour faire face aux changements climatiques. Deux cents soixante (260) personnes enquêtées, déterminées par échantillonnage de jugement ont participé à l'étude. Les outils d'investigation sont la recherche documentaire, l'observation et l'entretien. Quant à l'analyse des données, le mode de traitement est qualitatif et quantitatif. Au niveau des résultats, on retient que certaines cultures comme le maïs, le riz, le manioc, les aubergines, la tomate, etc., améliorées par le Centre National de Recherche Agronomique (CNRA) permettent aux cultivateurs dans la zone de Fresco par leur cycle de production court d'avoir non seulement accès au marché local, mais aussi de maximiser leurs revenus. Aussi, les associations culturales actuellement pratiquées dans cette zone favorisent l'amélioration de la productivité grâce à une utilisation optimale 
du sol et une protection des plantations grâce une couverture végétale prolongée.

Mots-clés: Résilience, changements climatiques, cultivateurs, association de culture, culture à court cycle

\title{
Analysis of Determinants of Resilience Climate Change of Fresco Department Cultivators, Ivory Coast
}

\author{
N'drin Owo Jean Arnaud, \\ Doctorant à l'UFR Anthropologie, \\ Université Félix Houphouët Boigny Cocody \\ Konan-Waidhet Arthur Brice, \\ Maître de Conférences, Télédétection et SIG, \\ UFR Environnement, Université Jean Lorognon Guédé \\ Kienon-Kabore Timpoko Hélène, \\ Professeure Titulaire en Histoire de l'Archéologie, Anthropologie, \\ Université Félix Houphouët Boigny Cocody
}

\begin{abstract}
Climate change is a global challenge facing all of humanity. Farmers, in addition to the many problems they already face, are now facing this new phenomenon. Adaptation currently seems to be one of the best alternatives. The analysis of this adaptation called resilience allows us to know the explanatory factors. In this perspective, the objective of this study is to analyse the climate change resilience of farmers in the Fresco department. Based on Bennett's theory of adaptive dynamics, the working hypothesis is that resilient farmers in Fresco department adopt short cycle crops and combine crops to cope with climate change. Two hundred and sixty (260) respondents, determined by judgment sampling, participated in the study. The investigative tools are documentary research, observation and interview. As for data analysis, the processing method is qualitative and quantitative. In terms of results, it is noted that some crops such as maize, rice, cassava, eggplants, tomatoes, etc., improved by the Centre National de Recherche Agronomique (CNRA) allow farmers in the Fresco area through their short production cycle to not only have access to the local market, but also to
\end{abstract}


maximize their income. In addition, the crop associations currently practiced in this area promote the improvement of productivity through optimal soil use and protection of plantations through extended vegetation cover.

Keywords: Resilience, climate change, farmers, crop association, short cycle crop

\section{Introduction}

\section{Le changement climatique, un phénomène universel}

Depuis le Sommet de la Terre au Brésil en 1992, la question du changement climatique et les graves risques qu'elle présente à la vie sur terre, ont poussé les gouvernements à travers le monde, à des degrés divers, à trouver les moyens de lutter contre la menace (SACC, 2014). Les politiques ont été nouées, les institutions ont été établies et les programmes ont été conçus à cet égard ${ }^{1}$.

Pourtant, une observation superficielle de la scène internationale montre que, par rapport à l'énormité et la gravité de l'enjeu, les progrès réalisés par les gouvernements ont été peu nombreux et espacés, et généralement limités et décevants (SACC, Id). Les émetteurs historiques n'ont pas réussi à tenir les engagements pris et les promesses de financement dans le cadre de responsabilités communes mais différenciées. Les pays en développement en général et les pays africains en particulier (en considérant que la région fait face aux plus grands risques) ont engagé les plus grands pollueurs dans les négociations internationales, mais les progrès ont été désespérément lents (Mbalamona, 2015). En tant que tel, la gouvernance internationale sur le climat a donné lieu à des actions éparses qui ont eu un effet contraignant direct sur la capacité des pays africains à faire face au changement climatique. Bien qu'émettant moins de $3 \%$ de Gaz à Effet de Serre (GES), l'Afrique n'échappe pas aux effets néfastes du changement climatique. Ils constituent une grande menace pour la croissance et le développement durable en Afrique, ainsi que pour la réalisation des Objectifs du Millénaire pour le Développement (OMD) (Mbalamona, op.cit.:1).

\footnotetext{
${ }^{1}$ Nous pouvons citer entre autres l'instauration d'un cadre mondial pour les services climatologiques, à l'initiative de l'Organisation des Nations Unis (ONU) et sous l'égide de l'Organisation Météorologique Mondiale (OMM), pour orienter le développement et l'utilisation d'informations et de services climatologiques scientifiquement fondés et faciliter ainsi la prise de décisions, et la Convention-Cadre des Nations-Unies sur le Changement Climatique (CCNUCC).
} 


\section{Les conséquences du changement climatique}

Ce phénomène provoque d'importants dégâts et perturbations dans de nombreux pays du continent notamment les inondations, les sécheresses, la modification des précipitations, l'assèchement des cours d'eau, etc., (CEA, 2011). À ce propos, selon le rapport du Cabinet britannique Maplecroft sur la vulnérabilité des pays et des villes au réchauffement climatique, publié en 2015, l'on enregistre sept (7) pays en Afrique sur les dix (10) pays les plus menacés, d'ici les trente prochaines années. Actuellement, les changements climatiques sont au centre des préoccupations aussi bien des acteurs scientifiques que des décideurs politiques au niveau mondial (Niang, 2009) car ils constituent un des nombreux obstacles au développement (Brown \& Crawford, 2008).

\section{Les approches pluridisciplinaires de l'étiologie des mesures d'adaptation}

Face à ces diverses situations, les besoins d'adaptations des populations aux changements climatiques apparaissent indispensables à leur survie (OCDE, 2009). Ces besoins d'adaptations sont diversement appréhendés par les auteurs. En effet, pour Clark (2006) les mesures d'adaptations les plus efficaces et durables sont souvent celles prises à l'échelle locale impliquant directement les personnes concernées. En outre, Dimon (2008) souligne que ces besoins d'adaptations développés par les cultivateurs en réponse aux conséquences négatives des changements climatiques dépendent de la perception et des connaissances endogènes qu'ils ont de ces changements. Aussi, Kanté (2011) soutient que l'adhésion des populations aux actions locales d'adaptations aux changements climatiques est effective si ces actions intègrent leurs savoirs endogènes. Cette approche est corroborée par Bambara \& al. (2013), qui soutiennent que les agriculteurs ont toujours pu faire face aux changements climatiques, générant au fil des ans des connaissances et un savoir-faire leur permettant de gérer leurs cultures. Cette adaptation aux changements climatiques appelée résilience n'est pas seulement une question de défi mais une réelle volonté de survie qui a pour fondement les savoirs endogènes des populations. Par ailleurs, les migrations et l'endettement sont également cités comme des stratégies d'adaptations réactives des populations face aux changements climatiques (IUCN, 2011 ; Kanté, op.cit.:1). Cependant, ces stratégies d'adaptations présentées par ces auteurs peuvent être considérées comme des conséquences compte tenu de leur connotation contraignante ; l'on s'endette ou l'on se déplace parce que l'on n'a pas le choix.

Il ressort de ces travaux que les perceptions et savoirs paysans sont devenus d'un intérêt majeur pour la compréhension et l'analyse de la résilience des populations face aux changements climatiques. 


\section{Les dispositifs de lutte contre les changements climatiques en Côte d'Ivoire}

Concernant la Côte d'Ivoire, en vue de lutter contre les changements climatiques, elle s'est dotée en 2014 d'une loi d'orientation sur le Développement Durable et en 2016 a pris un décret pour la mise en place dans toutes les organisations de la société, des Comités de Développement Durable. Les entreprises de Côte d'Ivoire ont activement participé à l'élaboration de la feuille de route de l'accord de Paris et se sont engagées à accompagner le gouvernement ivoirien à la mise en œuvre de cette feuille de route. En effet, les conséquences du changement climatique qui se font sentir dans tous les secteurs d'activité ont conduit les entreprises à aller vers des modes de production et de consommation durables. De nouvelles technologies sont testées et mises en œuvre. Des projets dans le domaine de l'atténuation, de la valorisation de la biomasse comme source d'énergie, la transformation des déchets en énergie, la mise en application de la Responsabilité Sociétale des Organisations, l'appui constant aux actions gouvernementales dans le cadre de la mobilisation des ressources financières pour la mise en œuvre du PND2016-2020. Les entreprises de Côte d'Ivoire ont des projets qui couvrent tous les domaines de l'accord de Paris. Elles s'engagent à contribuer de manière efficience à la mise en œuvre de l'accord de Paris. Par ailleurs, la Côte d'Ivoire s'est engagée à réduire ses émissions de GES de 28\% d'ici à 2030, pour contribuer à la lutte mondiale contre les changements climatiques. À cet effet, le Gouvernement ivoirien a traduit cet engagement au niveau national et international, dont la ratification de l'accord de Paris sur le Climat et des INDC (Intended Nationally Determined Contribution ou Contribution Prévue Déterminée au plan National), le Pays s'est doté d'une feuille de route (20162020) post CoP 21.

Sur le plan de l'agriculture, le Gouvernement ivoirien œuvre certes inlassablement à bâtir des stratégies climatiques nationales et à les mettre en harmonie avec le cadre international. Mais, force est de constater qu'en dépit de toutes ces initiatives, la détérioration continue des moyens d'existence qui entretient une pauvreté et une insécurité alimentaire chroniques pousse davantage les cultivateurs à la recherche de solutions adaptatives durables (Marema \& Crowley, 2014).

\section{La question et les objectifs de recherche}

Alors comment ces cultivateurs parviennent-ils à être résilients ? En d'autres termes, de quelles manières ces cultivateurs parviennent-ils à faire face aux défis des changements climatiques ? 
Les objectifs poursuivis par cette étude sont de deux (2) ordres :

- Décrire les perceptions paysannes des changements climatiques ;

- Déterminer le processus de la résilience aux changements climatiques des cultivateurs dans le département de Fresco.

\section{L'hypothèse et le cadre de référence théorique}

Pour atteindre ces objectifs, il sera procédé à la vérification de l'hypothèse suivante : Les cultivateurs résilients du département de Fresco adoptent des cultures à cours cycle et associent les cultures pour faire face aux changements climatiques.

La théorie des dynamiques adaptatives de Bennett (2005) qui propose une substitution du modèle systémique vers un modèle de systèmes et sous-systèmes interactifs dans lesquels les individus agissent stratégiquement en fonction de différentes rationalités, a été utilisée comme cadre de référence théorique. Cette théorie permet de voir les systèmes sociaux, quel que soit leur niveau de développement technologique, comme des mosaïques de sous-systèmes incorporant des normes et des valeurs différentes et antagonistes, avec de nombreux individus en mouvement entre celles-ci et les manipulant à des fins de gratification et de gain. Ceci implique que les acteurs locaux apprennent à se comporter de manière stratégique dans les différents sous-systèmes. Cette théorie permet de faire la lumière sur les différentes actions de la société pour répondre aux conditions imposées par les changements climatiques. Les stratégies adaptatives, les comportements adaptatifs et les actions stratégiques sont identifiés par l'application de cette théorie. La théorie des dynamiques adaptatives révèle aussi un caractère préventif, car elle met en relief les capacités de l'homme à s'adapter aux contraintes externes.

\section{Méthodologie}

\section{Sites et participants}

La présente étude s'est déroulée du 24 avril 2017 au 29 mai 2018 dans le département de Fresco, localisé entre les longitudes $5^{\circ} 29^{\prime}$ et $5^{\circ} 44^{\prime}$ Ouest et les latitudes $5^{\circ} 03^{\prime}$ et $5^{\circ} 11^{\prime}$ Nord. La ville de Fresco est située à $225 \mathrm{~km}$ à l'Ouest d'Abidjan ${ }^{2}$ et est limitée au Nord par le Département de Lakota, au Sud par l'Océan Atlantique, à l'Est par les Départements de Guitry et de Grand-Lahou et à l'Ouest par le Département de Sassandra. La population de Fresco est de 101298 habitants avec respectivement 53579 hommes contre 47719 femmes (RGPH, 2014). Les activités principales de la région sont l'agriculture et la pêche. Concernant l'agriculture, un accent particulier est mis sur les cultures pérennes procurant aux populations l'essentiel de leurs

\footnotetext{
${ }^{2}$ Capitale économique de la République de Côte d'Ivoire
} 
revenus. Les cultures pérennes pratiquées sont le cacao, le café et auparavant le coco dont les plantations étaient gérées par la COPAGRI. On note également le palmier à huile dont la culture est plus importante au Nord de la région, autour de l'usine Bolo et l'hévéa dont les surfaces cultivées s'accroissent de plus en plus. Les produits des plantations villageoises de palmier à huile, de coco et d'agrumes rencontrées dans la zone sont généralement destinés à l'autoconsommation ou au marché local.

Selon les services de l'Agence Nationale d'Appuis au Développement Rural (ANADER) et du Ministère de l'Agriculture et des Ressources Animales (MINAGRA) de Fresco, la tendance actuelle dans la zone est à l'abandon de la culture du café au profit de celles du cacao et de l'hévéa dont les produits sont pour l'instant mieux payés. Les plantations de café sont de ce fait abandonnées ou converties en plantations de cacao ou d'hévéa (l'hévéa qui est récent dans la zone et dont les superficies augmentent avec les années). Il existe dans le département de Fresco, 26 coopératives de producteurs de café et de cacao. Quant aux cultures vivrières, elles concernent le manioc, le riz, le maïs, la banane plantain et les maraîchers (aubergine, tomate, etc.). Le manioc et l'igname sont les cultures vivrières les plus pratiquées dans la zone. Le maïs et le riz sont principalement produits par les allogènes pour leur propre consommation. Les recherches dans le département de Fresco se sont déroulées précisément dans les villages de Zakaréko, Bohiko, Gbabam, Dahiri, Zegban et Yobéri. Ces différents sites ont été choisis du fait qu'ils nous sont apparus au cours de l'enquête préliminaire comme des sites susceptibles de nous permettre de mieux cerner notre objet d'étude. En effet, ces zones sont des sites qui ont une part active dans l'agriculture à Fresco. Ainsi, avec les cultivateurs de ces zones, nous avons pu recueillir des témoignages, avis et opinions sur les réalités du changement climatique dans le département de Fresco et les mesures adaptatives mises en place par ceux-ci pour lutter contre les effets de ce phénomène dans leur localité.

Le choix de la population d'étude, a obéi à un souci de diversification des sources de données. Ce sont au total deux cents soixante (260) personnes qui ont été interrogées. Cette population d'enquête a été obtenue à partir d'un échantillonnage par jugement. Ainsi, les groupes cibles suivants ont été retenus :

Tableau 1 : Répartition de l'échantillon

\begin{tabular}{|c|c|c|c|c|c|c|c|}
\cline { 2 - 7 } \multicolumn{1}{c|}{} & \multicolumn{6}{c|}{ Catégories sociales interrogées } \\
\hline Villages & $\begin{array}{c}\text { Cultivate } \\
\text { urs }\end{array}$ & $\begin{array}{c}\text { Commerça } \\
\text { nts }\end{array}$ & $\begin{array}{c}\text { Agents du } \\
\text { Ministère de } \\
\text { l'Agriculture } \\
\text { et des } \\
\text { Ressources } \\
\text { Halieutiques }\end{array}$ & $\begin{array}{c}\text { Coopérati } \\
\text { ves }\end{array}$ & $\begin{array}{c}\text { Autorités } \\
\text { G } \\
\text { administrati } \\
\text { ves ou } \\
\text { traditionnell } \\
\text { es }\end{array}$ & $\begin{array}{c}\text { Tot } \\
\text { al }\end{array}$ \\
\hline Zakaréko & 30 & 10 & 2 & 7 & 2 & 5 & 56 \\
\hline
\end{tabular}




\begin{tabular}{|c|c|c|c|c|c|c|c|}
\hline Bohiko & 20 & 9 & 2 & 5 & 3 & 6 & 45 \\
\hline Gbabam & 19 & 10 & 1 & 2 & 1 & 1 & 34 \\
\hline Dahiri & 22 & 5 & 2 & 4 & 2 & 4 & 39 \\
\hline Zegban & 30 & 9 & 3 & 4 & 3 & 3 & 52 \\
\hline Yobéri & 15 & 5 & 2 & 5 & 2 & 5 & 34 \\
\hline Total & $\mathbf{1 3 6}$ & $\mathbf{4 8}$ & $\mathbf{1 2}$ & $\mathbf{2 7}$ & $\mathbf{1 3}$ & $\mathbf{2 4}$ & $\mathbf{2 6 0}$ \\
\hline
\end{tabular}

Source : Enquêtes 2018

\section{Instruments de recueil des données}

La collette des informations relatives à l'objet d'étude s'est faite à partir de la recherche documentaire, de l'observation et de l'entretien.

\section{- Recherche documentaire}

La recherche documentaire a consisté à consulter des ouvrages aux services des archives et de documentation de certaines institutions, telles que : le Centre de Recherches et d'Actions pour la Paix, le centre culturel français, la bibliothèque de l'ambassade des États-Unis d'Amérique. Sur internet, des articles, des rapports de recherches d'organismes internationaux, des thèses et mémoires établis dans d'autres universités, ont été parcourus. La recherche documentaire, bien qu'ayant été très utile dans nos recherches, a montré certaines limites que d'autres instruments de recueil et de collecte des données ont corrigées, tels que l'observation et l'entretien.

\section{- Observation}

L'observation dans la résilience, joue un rôle prépondérant dans l'identification de la particularité des comportements d'un sujet résilient ou en plein processus de résilience. Ainsi, nous avons fait appel à l'observation systémique, «qui est une technique, qui permet de décrire et d'identifier les interactions » (Ciccone, 1998) entre les cultivateurs et leur milieu de vie. Cette observation qui s'est faite essentiellement dans les villages de Zakaréko, Bohiko, Gbabam, Dahiri, Zeban et Yobéri a duré treize (13) mois. Elle s'est faite à l'aide d'une grille d'observation qui a permis d'analyser des comportements, pratiques et techniques culturales des cultivateurs dans le département de Fresco.

\section{- Entretien}

L'étude est principalement axée autour du vécu des cultivateurs du département de Fresco exposés aux changements climatiques. Elle s'est donc intéressée à recueillir les opinions des enquêtés sur les attitudes développées face à la situation d'exposition aux changements climatiques. Des entretiens semi-directifs ont eu lieu dans les villages de Zakaréko, Bohiko, Gbabam, Dahiri, Zegban et Yobéri. Ils ont concerné les coopératives, les agents du conseil général, les cultivateurs, les commerçants, les ONG, les autorités 
administratives ou traditionnelles (Chef de village, leader de jeunesses, etc.,). Les agents du Ministère de l'Agriculture et des Ressources Halieutiques ont été également interviewés. Interroger ces personnes nous paraissait nécessaire parce que la résilience des cultivateurs exposés aux changements climatiques implique tous ces acteurs. Nous avons donc élaboré trois (3) différents guides d'entretien afin d'échanger avec ces différentes catégories de personnes et d'évaluer l'influence des pratiques et techniques culturales dans le processus de résilience. Les axes thématiques abordés par ces différents guides d'entretien sont les manifestations des changements climatiques, les baisses des productions, l'adoption de nouvelles cultures, le rendement des nouvelles cultures, les aides des coopératives et du Gouvernement, etc.

\section{Méthodes d'analyse des données}

S'agissant du mode de traitement des données, deux (2) types d'analyse ont été effectués : l'analyse quantitative et l'analyse qualitative. L'analyse quantitative a permis la mesure ou le regroupement des données issues des questionnaires à l'aide de l'outil informatique. Pour mener à bien cette analyse, le logiciel informatique Statistical Package for the Social Sciences version 20 a été utilisé. Quant à l'analyse qualitative, elle a permis de mieux comprendre le vécu des populations en termes de pratiques, significations, perceptions du phénomène étudié et de cerner les logiques des acteurs. Elle a donc permis d'analyser les données relatives aux avis, opinions, discours et idées exprimées par les personnes interviewées.

\section{Résultats}

L'exposé des résultats s'articule autour de deux (2) axes, notamment les perceptions paysannes des changements climatiques et le processus de la résilience des cultivateurs du département de Fresco exposés aux changements climatiques.

\section{Perceptions paysannes des changements climatiques}

L'étude des perceptions paysannes des changements climatiques apparait importante car l'adhésion des populations aux actions locales d'adaptation aux changements climatiques est relative à leurs savoirs endogènes. Ce point vise ainsi à connaître les visions paysannes des manifestations locales des changements climatiques et leurs conséquences socio-environnementales et d'apprécier l'ampleur des perceptions des indicateurs de changements relevés. Selon les personnes enquêtées, les facteurs climatiques considérés sont de deux (2) catégories notamment les facteurs cosmiques et géographiques. Ces facteurs climatiques sont appréciés à travers un ensemble de paramètres climatiques et de leurs indicateurs. Le tableau ci-dessous en fait l'exposé : 
Tableau 2 : Paramètres climatiques et indicateurs des changements cités par les cultivateurs

\begin{tabular}{|l|l|}
\hline \multicolumn{1}{|c|}{ Paramètres climatiques } & \multicolumn{1}{c|}{ Indicateurs des changements } \\
\hline \multirow{4}{*}{ Pluviométrie } & - Diminution de la rosée \\
& - Arrêt précoce des pluies \\
& - Retard des pluies \\
& - Fréquence des poches de sécheresse \\
& - Agressivité des pluies \\
& - Sécheresse de l'air ambiant \\
& - Fréquente alternance inondation/sécheresse \\
& - Fréquence d'apparition d'arc-en-ciel en début \\
& de saison pluvieuse \\
& - Pluviosité en baisse \\
& - Raccourcissement de la durée de la saison \\
& pluvieuse \\
\hline \multirow{3}{*}{ Température } & - Fréquence de l'avortement des pluies \\
\hline \multirow{3}{*}{ Vent } & - Températures de plus en plus chaudes \\
& - Fraicheur en début de saison pluvieuse \\
& - Froid intense \\
\hline & - Vents de plus en plus violents \\
& - Augmentation des tourbillons \\
& - Fréquence de brouillard de poussière \\
& - Apparition de tourbillons en saison pluvieuse \\
\hline
\end{tabular}

Source : Enquêtes, 2018

\section{Perceptions paysannes concernant les indicateurs des changements climatiques}

Les perceptions paysannes concernant les indicateurs des changements climatiques seront analysées autour trois (3) principaux paramètres: la pluviométrie, la température et le vent.

\section{Pluviométrie}

Dans les différentes zones enquêtées, les indicateurs des changements pluviométriques tels que l'arrêt précoce des pluies, le raccourcissement de la durée de la saison pluvieuse, la fréquence alternance inondations /sécheresse, pluviométrie en baisse, le retard des pluies et la fréquence des poches de sécheresse ont été reconnus par près de $100 \%$ des cultivateurs interviewés. Les taux de perception variant entre $80-90 \%$ des cultivateurs interviewés ont les indicateurs suivants, la fréquence de l'avortement des pluies et l'agressivité des pluies. Les taux de perception de moins de 50\% des cultivateurs interviewés présentent les indicateurs pluviométriques comme la diminution de la rosée, la fréquence d'arc-en-ciel en début de saison pluvieuse, la sécheresse de l'air ambiant. L'ensemble des données recueillies présentent l'importance des changements des précipitations dans les zones enquêtées (Figure 1). 


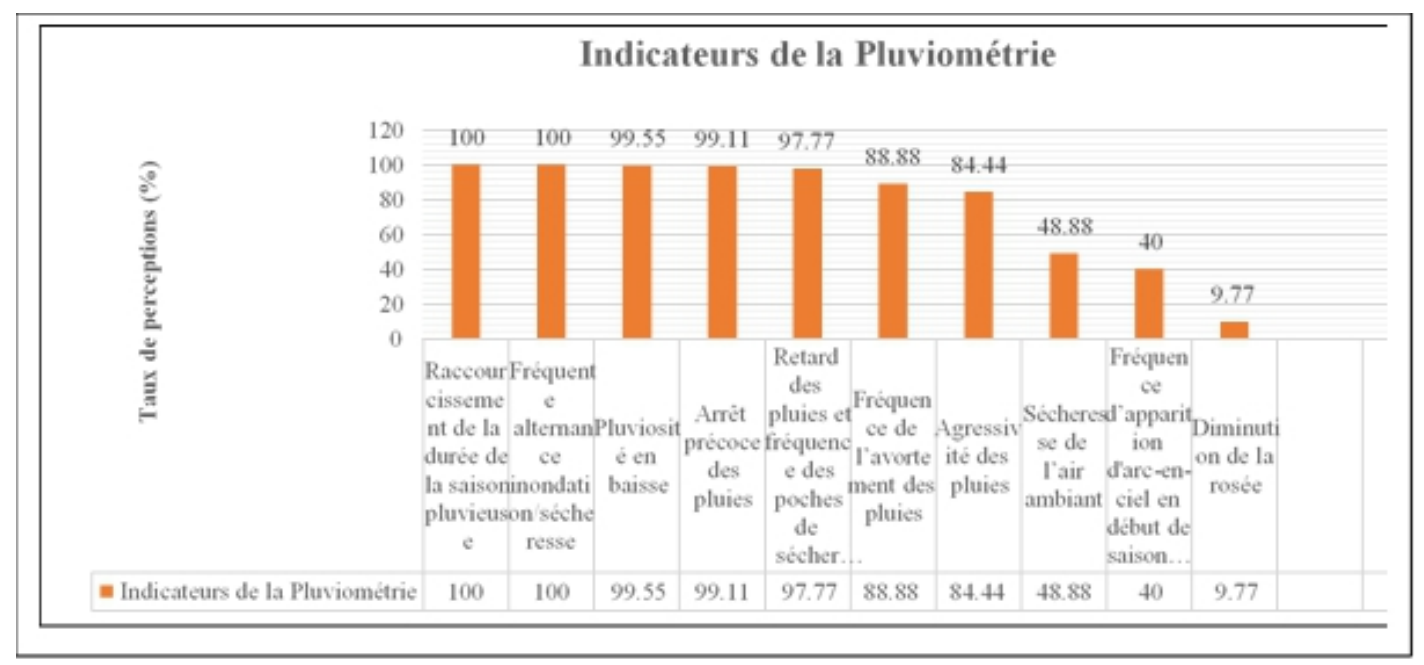

Figure 1 : Indicateurs de la Pluviométrie selon les personnes enquêtées

Source : Enquêtes, 2018

\section{Température}

Les indicateurs liés à la température ont été diversement appréciés par les cultivateurs enquêtés dans les différentes zones d'étude. Les indicateurs de changements de température sont : les températures de plus en plus chaudes, la fraîcheur en début de saison pluvieuse et le froid intense. L'indicateur de la fraîcheur en début de saison pluvieuse reconnu par 98,22\% des cultivateurs enquêtés apparait comme l'indicateur le plus élevé. 93,34\% des cultivateurs interrogés ont signalés avoir observés que les températures deviennent de plus en plus chaudes. Moins de 50\% des personnes enquêtées ont présenté des taux de perceptions relativement bas à savoir le froid intense (Figure 2).

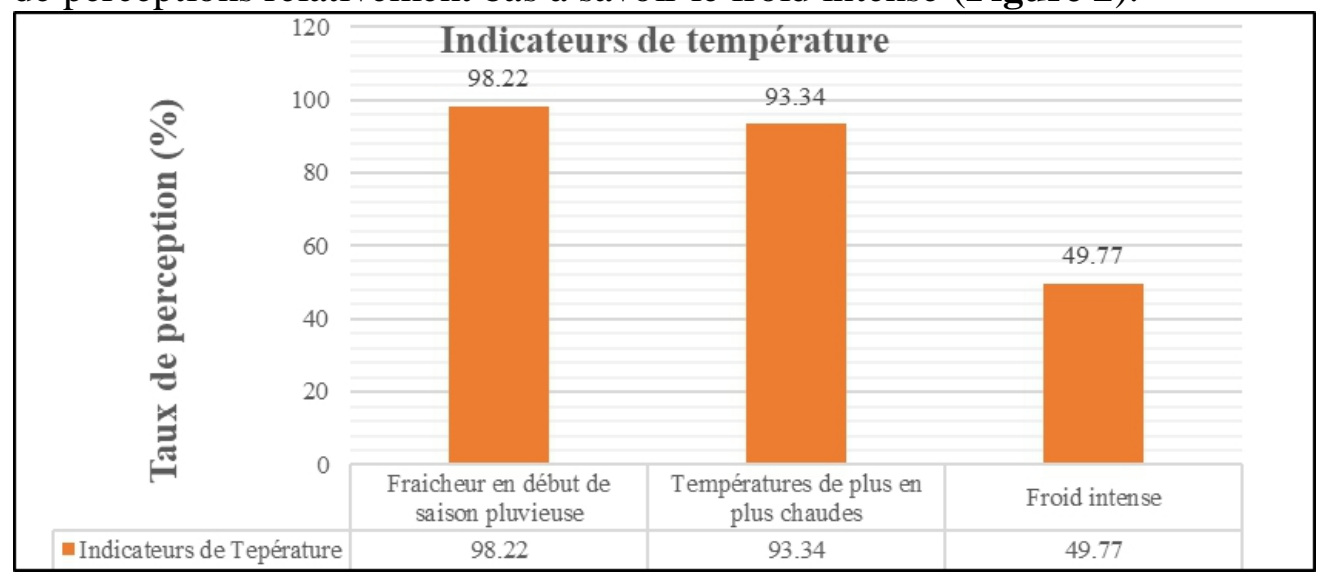

Figure 2 : Perceptions des changements liés à la température

Source : Enquêtes, 2018 


\section{Vent}

Les vents deviennent de plus en plus violents selon 47,55\% des cultivateurs enquêtés Tandis que $11,11 \%$ ont signalé l'augmentation des tourbillons. Enfin, respectivement $9,77 \%$ et $4,44 \%$ ont relevé la fréquence de bouillards de poussière et l'apparition de tourbillons en saison pluvieuse (Figure 3).

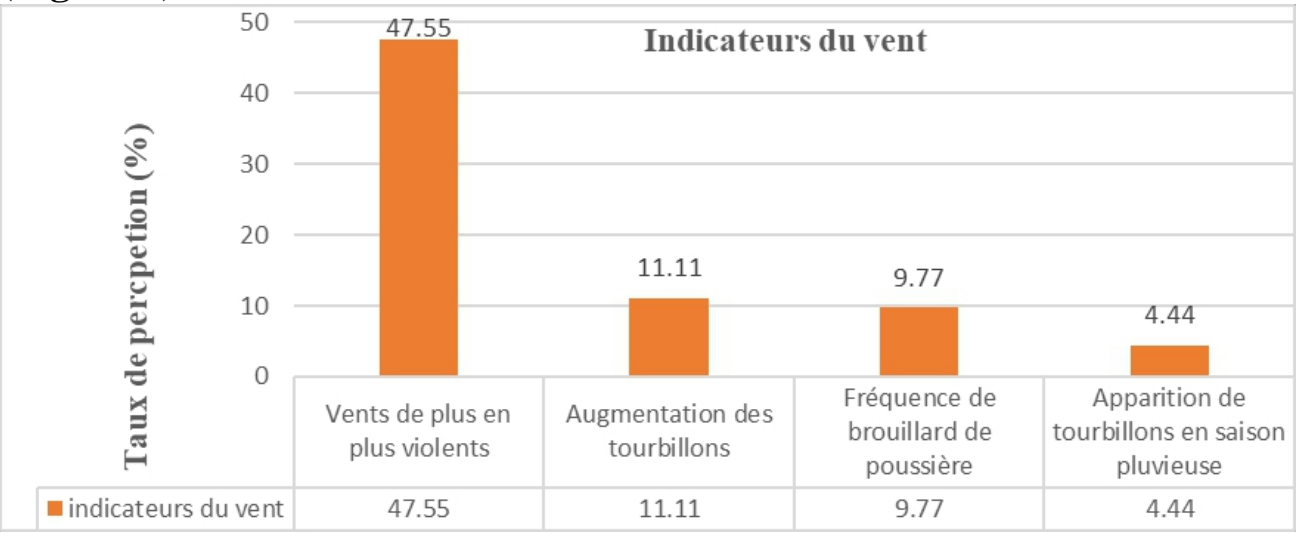

Figure 3 : Perceptions des changements liés au vent

Source : Enquêtes, 2018

Perceptions paysannes des effets des changements climatiques sur l'agriculture et les populations

Les changements climatiques affectent sérieusement la disponibilité des ressources naturelles telles que l'eau, la terre, etc., qui laisse de graves impacts sur l'agriculture et les populations. Le Tableau (3) présente les données relatives aux effets des changements climatiques perçus par les cultivateurs :

Tableau 3 : Effets des changements climatiques selon les enquêtés

\begin{tabular}{|cl|c|c|c|}
\hline & Fréquence & Pourcentage & Pourcentage valide \\
\hline Valide & Baisses de rendements agricoles & 74 & 28,45 & 28,46 \\
& Inondation villageoise & 65 & 25 & 25 \\
& Modifications pédologiques & 50 & 19,23 & 19,23 \\
& Exploitation abusive de la forêt & 36 & 13,85 & 13,85 \\
& Modification de la culture alimentaire & 25 & 9,61 & 9,61 \\
& Migration & 10 & 3,84 & 3,85 \\
& Total & 260 & 99,98 & 100,0 \\
Manquant & Système & 0 & 0 & \\
Total & & 260 & 100,0 & \\
\hline
\end{tabular}

Source : Enquêtes, 2018

Les données du Tableau (3) présentent les perceptions des personnes interrogées sur les effets des changements climatiques dans le département de Fresco. Selon elles, les changements climatiques entrainent : les modifications 
pédologiques $(19,23 \%)$, les baisses de rendements agricoles (28,46\%), l'exploitation abusive de la forêt $(13,85 \%)$, les modifications de la culture alimentaire $(9,61 \%)$, la migration $(8,89 \%)$ et les inondations villageoises $(25$ $\%)$. Il ressort en général de ce tableau que les changements climatiques ont divers effets sur l'agriculture et les populations. Les plus significatifs sont les baisses des rendements et les inondations villageoises qui totalisent comme pourcentage $53,46 \%$ des données recueillies. Ces éléments d'analyse permettent de se rendre compte que les effets négatifs des changements climatiques touchent sérieusement aux sources de revenus et à l'habitat des populations, déjà vulnérables à causes de leurs conditions de vie précaires (pauvreté, taille importante de la famille, déscolarisation, etc.).

\section{Modifications pédologiques}

Les constats faits dans les zones étudiées font état de la diminution de la fertilité des sols. Cette diminution est provoquée par les pertes improductives de nutriments (par infiltration, érosion ou dans l'atmosphère) et par l'extraction des nutriments. Cette modification pédologique est un problème majeur dans la région de Fresco à en croire les personnes enquêtées. Nous corroborons ces affirmations par les dires des cultivateurs exposés aux changements climatiques qui ont été enquêtés : «Avant la terre était de couleur noire forcée, humide et bien compacte. Nos cultures se développaient très bien. Et le travail de la terre n'était pas difficile parce le bon développement des plantes, nous encourageait à travailler la terre. Mais ces dernières années, je vous affirme que le travail de la terre n'a jamais été aussi difficile. Le sol est devenu sec, dure à travailler et les récoltes sont de mauvaises qualités», propos de P.F, un cultivateur. «La vérité est que nous sommes piégés par la terre. Car en dehors du travail de la terre, nous $n$ 'avons aucune autre qualification professionnelle. Nos parents nous ont appris le travail de la terre pour subvenir aux besoins de nos familles. Mais les fruits de la terre n'arrivent plus à survenir à nos besoins. La terre a changé de couleur et elle est de plus en plus pauvre», propos de D.C., un cultivateur. «Nous n'avons pas besoin de spécialiste pour savoir que la terre a connu des modifications dans cette région. À vrai dire le meilleur indicateur pour savoir cela, ce sont les récoltes qui perdent en quantité et en qualité», propos de J.B., un agent du Conseil Général de Fresco. Ces propos de nos enquêtés apportent la preuve des modifications pédologiques constatées dans les zones enquêtées. Ainsi, nous pouvons présumer que l'allongement de la saison sèche comme conséquence des changements climatiques dans ces zones entraine le dessèchement et la dureté des sols et impacte davantage négativement le développement des cultures mais particulièrement les récoltes. 


\section{Baisses de rendements agricoles}

Les données recueillies auprès des personnes enquêtées présentent également une autre conséquence des changements climatiques. En effet, les changements climatiques à travers leurs effets néfastes affectent profondément l'agriculture dans la région de Fresco. Or pour ces cultivateurs l'agriculture est la principale source de leurs revenus et leur premier mode de subsistance. Selon ces derniers, les baisses de rendements sont dues aux pertes de récoltes considérables, aux mauvaises qualités de produits récoltés, etc. Nous corroborons ces affirmations par les dires des cultivateurs exposés aux changements climatiques qui ont été enquêtés : "Avant avec une récolte, je pouvais gagner jusqu'à $500.000 \mathrm{f}$. Et cette somme me permettait de prendre en charge les frais de scolarité de mes enfants et le reste de ma famille. Mais aujourd'hui, les récoltes sont tellement maigres que la vente des produits récoltés ne couvre que les dépenses pour la main d'œuvre et les produits. Après ça, le peu qu'il reste ne sert qu'à couvrir seulement les dépenses de la famille. Je suis obligé de m'endetter pour assurer la scolarité de mes enfants. À cette allure qu'est-ce qu'on va devenir ? », propos de D.A., un cultivateur de Fresco. "Vous savez le plus dure c'est que non seulement les récoltes sont de petites quantités, mais elles sont aussi de mauvaises qualités. Par exemple, les fèves de cacao sont de plus en plus petites. Les tubercules de manioc sont de très petites tailles », propos de K.L., un cultivateur de Fresco. Ces propos de nos enquêtés font état des conséquences vécues par ceux-ci à travers la baisse de leur revenu due en partie par la baisse de rendements de leurs cultures. Cette baisse des revenus des cultivateurs de Fresco fragilise davantage leur condition de vie et les confine de plus en plus dans une situation de précarité qui se traduit surtout par la limitation de leur capacité d'accès aux ressources et services.

\section{Exploitation abusive de la forêt}

D'une manière générale, l'amenuisement des revenus pousse les cultivateurs à l'intensification des surfaces cultivées, c'est-à-dire à l'investissement de travail et de capital pour augmenter la production. Aussi, avec l'accentuation de l'aridité et des aléas climatiques qui l'accompagnent, les cultivateurs optent pour une extensification de leurs cultures. Cette pratique apparait ainsi comme une mesure adaptative pour augmenter leurs revenus. Nous confirmons ces affirmations par les dires des cultivateurs exposés aux changements climatiques qui ont été enquêtés : «les récoltes sur nos anciennes parcelles devenant de plus en plus insuffisantes, nous avons choisi de cultiver de nouvelles parcelles de manière à ce que les anciennes et les nouvelles nous permettent de gagner mieux notre vie. C'est tout ce qui nous reste à faire. C'est vrai qu'augmenter la taille de nos parcelles revient à travailler davantage mais nous sommes obligés de le faire », propos de T.M., 
un cultivateur de Fresco. "C'est dans le travail que se trouve notre salut. Les nouvelles réalités climatiques menacent la survie des populations. Ainsi, pour faire face à cette dure réalité du climat, elles choisissent d'exploiter davantage la forêt. Cette mesure pourrait être qualifiée d'inadéquate et nuisible pour la forêt mais essayons de les comprendre, les populations sont désespérées »», propos de M.L, un agent d'une ONG de lutte contre la déforestation. À travers ces propos de nos enquêtés, nous comprenons que le recours à la forêt pour agrandir les espaces cultivées apparait comme un moyen de lutte contre les effets néfastes des changements climatiques. La flore et la faune des forêts dans la région de Fresco sont extrêmement variées. Le maintien de ces vastes étendus de forêts représentatives de tous les écosystèmes reste une préoccupation des autorités ivoiriennes face à une demande des produits forestiers de plus en plus croissante.

\section{Inondation villageoise}

Les observations menées sur les effets néfastes des changements climatiques à Fresco indiquent l'existence de phénomènes météorologiques extrêmes, notamment les inondations. En effet, les inondations dans cette zone sont devenues de plus en plus fréquentes, avec des conséquences dramatiques sur l'environnement, l'économie, les établissements humains et la vie humaine. Elles s'expliquent par le fait que ces localités urbaines et rurales de Fresco présentent pour la plupart, un environnement fortement dégradé sous les effets conjugués des aléas climatiques. Ainsi, plusieurs populations se sont retrouvées dans des zones qui sont en réalités des lits majeurs inondables. On constate également que ces zones sont à risque du fait de l'inexistence d'ouvrages pour l'assainissement des eaux usées et pluviales. Nous confirmons ces affirmations par les dires des cultivateurs exposés aux changements climatiques qui ont été enquêtés : «Nous sommes venus occuper ce site à la suite de récurrents conflits avec nos anciens voisins. Mais depuis que le village s'est installé ici, nous avons ces dernières années été victimes de plusieurs inondations. C'est avec l'aide du Conseil Général et de la Mairie que des caniveaux et des murs ont été réalisés pour drainer l'eau de pluie et les eaux usées pour mieux protéger notre village. Aujourd'hui, on est toujours victimes d'inondations mais elles sont moins catastrophiques que celles $d u$ passé ou il y avait eu des morts d'hommes »», propos de B.T., un cultivateur de Fresco. «Le nouveau climat nous fatigue vraiment. En plus de rendre la terre aride et pauvre pendant les saisons sèches, nos villages sont constamment inondés pendant la saison pluvieuse. En plus de voir nos villages inondés, les bas-fonds où on cultive le riz par exemple sont constamment inondés. Les conséquences de ces inondations sont de plus en plus graves avec les plantations détruites, les récoltes et les vies humaines perdues », propos de M.C., un cultivateur de Fresco. À travers ces propos de nos enquêtés, il 
ressort que les cultivateurs sont fréquemment victimes d'inondations. Ces inondations touchent toutes les zones basses à l'intérieur des villes et des villages : fonds de vallées, cuvettes et terrains plats, etc. Ainsi, pendant la saison des pluies, les eaux inondent de nombreuses habitations construites dans ces zones. Des manifestations catastrophiques de ce type de phénomène se sont toujours produites dans beaucoup de villages, avec plus d'acuité dans ceux situés à proximité de cours d'eaux (lagunes, etc.).

\section{Modification de la culture alimentaire}

La diminution des récoltes pousse les cultivateurs à rechercher d'autres variétés culturales plus propices à la variation du climatique, c'est-à-dire des plantes qui produisent plus et moins contraignantes. Le défi est non seulement d'augmenter la production mais aussi d'assurer la sécurité alimentaire. Cette sécurité alimentaire a été mise en mal par les aléas climatiques aux dires des enquêtés, entrainant ainsi des modifications alimentaires notamment l'adoption de nouveaux régimes alimentaires rendue possible avec les nouvelles variétés culturelles. Nous confirmons ces affirmations par les dires des cultivateurs exposés aux changements climatiques qui ont été enquêtés : «Maintenant nos habitudes alimentaires ont beaucoup changé avec la variation des saisons. Par exemple, l'attiéké est devenu notre aliment de base. Il est associé à plusieurs aliments comme le poisson et la viande », propos de Y. L., un cultivateur de Fresco. "Il est évident que les cultures qui s'adaptent le mieux au nouveau climat sont celles que les cultivateurs privilégient soit pour le commerce soit pour leur alimentation. Partant de ce fait, certaines cultures jadis secondaires sont devenues les plus cultivées et consommées. Ces modifications alimentaires sont la conséquence directe des changements climatiques », propos de B.N., un agent de coopérative de Fresco. À travers ces propos de nos enquêtés, nous pouvons affirmer que les changements climatiques engendrent comme conséquence des modifications alimentaires dues en partie aux nouvelles pratiques culturales adoptées par les cultivateurs pour faire face aux variations climatiques.

\section{Migration}

Les constats réalisés dans certains villages ont montré que certaines populations ont à cause des conséquences négatives des changements climatiques choisi de migrer, de se déplacer vers d'autres zones jugées plus propices à leurs activités agricoles. Ainsi, nous pouvons affirmer que cette migration résulte de leur incapacité à s'adapter aux nouvelles réalités climatiques. Nous confirmons ces affirmations par les dires des cultivateurs exposés aux changements climatiques qui ont été enquêtés : "Certains de nos amis ou voisins ont quitté le village pour aller s'installer ailleurs. Certains jeunes ont également quitté notre village pour la ville. Leur départ se 
comprend car la terre qui était la source de leur espoir pour bâtir une famille heureuse est devenue de plus en plus pauvre. Ils ont fourni beaucoup d'efforts à travailler cette terre pour finalement avoir de maigres récoltes », propos de G.S., un cultivateur de Fresco. "J'ai quitté mon village pour venir me débrouiller à la gare des taxis motos. Avant, je cultivais le riz avec mon père mais avec la sècheresse et les inondations, cultiver le riz n'est plus rentable comme par le passé. J'avais un peu d'argent et j'ai emprunté une somme d'argent pour acheter mon taxi-moto. Aujourd'hui, je suis dans le transport et je gagne bien ma vie. Seulement mes parents et mon village me manquent », propos de F.L., un conducteur de Fresco. "Il y a des villages entiers qui ont été déplacés avec les inondations qui tuent des villageois. Ces déplacements forcés sont provoqués par les changements climatiques », propos de K.A., un cultivateur de Fresco. À travers ces propos de nos enquêtés, nous retenons que les déplacements de populations dans la région de Fresco sont dus, d'une part, à la dégradation des conditions d'existence de ces populations et à la menace sur la sécurité alimentaire et, d'autre part, à l'accroissement de la population entrainant une compétition plus accrue pour des ressources qui se raréfient.

\section{Processus de la résilience des cultivateurs exposés aux changements climatiques \\ Manifestations de la résilience}

Parler de résilience en termes de résultat revient à la considérer comme la conséquence de tels ou tels facteurs. La résilience ne s'observant que par un ensemble de comportements définis au préalable. En effet, toute recherche empirique se doit de définir des critères diagnostiques de résilience, et ceci pour pouvoir distinguer les comportements résilients des comportements non résilients. Ainsi la résilience des cultivateurs exposés aux changements climatiques se constate principalement dans ses effets, notamment, l'adoption de cultures à cours cycle et l'association de cultures.

\section{Facteurs explicatifs de la résilience des cultivateurs exposés aux changements climatiques}

De façon générale, plusieurs raisons sont avancées pour expliquer la résilience des cultivateurs exposés aux changements climatiques dans le département de Fresco. Il s'agit de l'adoption de cultures à cours cycle et l'association de cultures. Le Tableau (4) présente les différents pourcentages des assertions d'enquêtés concernant les facteurs qui contribuent à la résilience des cultivateurs exposés aux changements climatiques dans le département de Fresco. 
Tableau 4 : Présentation des facteurs explicatifs et leurs pourcentages

\begin{tabular}{|c|c|c|}
\hline Facteurs explicatifs & Effectifs & Pourcentages (\%) \\
\hline Adoption de cultures à cours cycle & 163 & 62,69 \\
\hline Association des cultures & 97 & 37,31 \\
\hline Total & 260 & 100 \\
\hline
\end{tabular}

Source : Enquêtes, 2018

Il ressort de l'analyse des données du Tableau 4 que 37,31\% des personnes enquêtées attestent que l'adoption de cultures à cours cycle explique la résilience des cultivateurs exposés aux changements climatiques dans le département de Fresco. Par ailleurs, 62,69\% des personnes enquêtées affirment pour leur part que la résilience des cultivateurs exposés aux changements climatiques dans le département de Fresco a favorisée l'association des cultures. Ces données prédisent une relation entre la résilience des cultivateurs exposés aux changements climatiques et l'adoption de cultures à cours cycle et l'association de cultures. Dans le souci de confirmer la relation de causalité qui semble exister entre ces deux variables, nous avons procédé à un test statistique. Ainsi, pour cet exercice notre choix s'est porté sur le test du $\mathrm{Khi}^{2}$. Ces différentes variables étant des variables qualitatives, l'utilisation du test du $\mathrm{Khi}^{2}$ est plus appropriée pour apprécier le lien existant entre ces différentes variables. Pour ce faire, nous considérons :

L'hypothèse alternative, H1: Il existe un lien entre la résilience des cultivateurs exposés aux changements climatiques et l'adoption de cultures à cours cycle et l'association de cultures.

L'hypothèse nulle, HO : Il n'existe pas de lien entre la résilience des cultivateurs exposés aux changements climatiques et l'adoption de cultures à cours cycle et l'association de cultures.

Le résultat du $\mathrm{Khi}^{2}$ de 3,882 est significatif au seuil de probabilité 0,05 avec un degré de liberté 1 . Le Khi² calculé est supérieur au Khi² lu : 3,882> 3,841, donc $\mathbf{H 0}$ est rejetée.

L'hypothèse de travail se trouve ainsi vérifiée. À la vue des résultats, nous avons un lien significatif entre la résilience des cultivateurs exposés aux changements climatiques et l'adoption de cultures à cours cycle et l'association de cultures. Pour connaitre l'intensité de ce lien, nous calculerons le $\mathrm{V}$ de Cramer $(\mathbf{0}>\mathbf{V}>\mathbf{1})$. Selon les résultats, le V de Cramer est de 0,67; donc le lien existant est relativement très fort. En d'autres termes, la résilience des cultivateurs exposés aux changements climatiques s'explique par l'adoption de cultures à cours cycle et l'association de cultures. 


\section{Adoption de cultures à cours cycle}

Les cultivateurs à Fresco ont adopté, des pratiques visant à réduire les effets négatifs des aléas climatiques notamment les inondations, les tempêtes, les glissements de terrains, les sécheresses et canicules et les feux de brousse, la dégradation des terres, la baisse du débit des fleuves et des hauteurs des pluies, l'érosion côtière, etc. Parmi celles-ci, on peut citer la pratique du semis précoce et l'utilisation de cultures à cycle court. La pratique de l'adoption de cultures à cours cycle a l'avantage de faire coïncider au mieux le cycle de la culture avec la saison des pluies, et de réduire ainsi la période de stress hydrique en fin de cycle. L'adoption de cultures à cours cycle constitue une pratique très répandue à Fresco. Ses bénéfices sont nombreux. En effet, elle assure une couverture de la superficie cultivée et elle augmente à la fois la productivité de la terre (biomasse) et celle du travail. Cette méthode apparait ainsi comme une stratégie d'adaptation face à l'accentuation des risques climatiques qui se précise selon les experts de jour en jour.

Les produits tirés de cette pratique culturale servent aussi bien à l'autoconsommation qu'à l'obtention de revenus monétaires. C'est le cas notamment des cultures vivrières dont les apports constituent une part importante dans l'alimentation et l'économie locale des populations de Fresco. En effet, selon une étude menée par le Ministère de l'Agriculture et du Développement Rural (MINADER, 2006), les cultures vivrières constituent la base de l'alimentation humaine avec $36.2 \%$ des apports calorifiques et $40 \%$ des apports protéiniques, dont $19.5 \%$ et $22 \%$ pour le maïs. Ces données soulignent ainsi le rôle prépondérant de ce type de culture pour les populations. Mais les cultures vivrières il y a de cela quelques décennies n'occupaient pas une telle importance dans l'agriculture à Fresco. En effet, dans cette zone la tendance était plus la culture du café, du cacao et récemment l'hévéa dont les produits étaient les mieux payés. Cependant, avec les incessantes fluctuations du prix de ces cultures pérennes et des nombreuses maladies qui déciment ces plantes, certains cultivateurs ont abandonné ou converti les plantations de Cacao, de café en plantations de vivriers. Ces cultures vivrières concernent le manioc, le riz, le maïs, la banane plantain et les maraîchers (aubergine, tomate, etc.). Le manioc et l'igname sont les cultures vivrières les plus pratiquées dans la zone. Le maïs et le riz sont principalement produits par les allogènes pour leur propre consommation.

Nous confirmons ces affirmations pas les dires de certains responsables du CNRA et des cultivateurs exposés aux changements climatiques qui ont été enquêtés : "Créé en 1998, le Centre National de Recherche Agronomique (CNRA) est une société anonyme à participation financière publique minoritaire. Il a pour mission de mener des recherches et d'en diffuser les résultats, de conserver et de valoriser son patrimoine scientifique 
CNRA intervient principalement dans les domaines agricoles et agroindustriels à savoir, systèmes de production, productions végétales, animales et forestières, innovations technologiques, méthodes de conservation et de transformation. Ce répertoire de variétés améliorées permettra aux producteurs de choisir les variétés à cultiver en fonction de leurs caractéristiques agronomiques, technologiques et de leurs qualités organoleptiques. Le but de son centre est de lutter contre l'insécurité alimentaire et nutritionnelle en Côte d'Ivoire, en aidant les cultivateurs», propos du Directeur général du CNRA, Yté Wongbé., " les semences, notamment celles de variétés améliorées, constituent un intrant primordial pour accrô̂tre la productivité agricole. Elles sont essentielles lans les systèmes agricoles et constituent la première étape vers la production d'aliments pour assurer la sécurité alimentaire des populations. Le succès des agriculteurs dans leurs activités de production dépend de la qualité, de l'accessibilité et la diversité des semences. Le répertoire variétal regroupe les fiches descriptives des variétés améliorées de cultures vivrières notamment du riz, du maïs, du plantain, de l'igname, du manioc, du gombo, du piment, de l'aubergine, du soja, du niébé que nous avons mis à la disposition des cultivateurs», propos du Dr Louise Akanvou, responsable du département ressources génétiques du CNRA. «Grâce au CNRA, nous avons eu des semences améliorées de mais, de manioc, de l'igname et bien d'autres cultures qui nous permettent d'augmenter nos productions. Aussi les responsables du CNRA nous ont montré des techniques pour cultiver sur une même parcelle des plantes qui produisent à différentes périodes», propos de D.D. un cultivateur à Fresco. «Avant on produisait que le cacao et le café. Mais nous avons vu nos récoltes diminuées avec les maladies du cacao et du café. Les agents du CNRA venus pendant une campagne dans notre village, nous ont montré de nouvelles techniques pour produire toujours. Les variétés améliorées du CNRA sont plus résistantes aux changements du climat et produisent plus. À travers ces propos de nos enquêtés, il ressort que certaines cultures comme le maïs, le riz, le manioc, les aubergines, la tomate, etc., améliorées par le CNRA permettent aux cultivateurs dans la zone de Fresco par leur cycle de production court d'avoir non seulement accès au marché local mais aussi de maximiser leurs revenus. Ainsi, peut-on dire que l'adoption de cultures à cours cycle constituent un espoir pour les populations car non seulement elles nourrissent l'être humain, du nourrisson à l'adulte, sous différentes formes de cuisson, mais aussi elles servent d'aliments pour les animaux (bétail, volailles, etc.,) et elles fournissent des dérivées de produits dans les fabriques industrielles et artisanales (savon, attiéké, huile, pommade, etc.). 


\section{Association de cultures}

Les observations sur le terrain ont montré que les cultivateurs afin de pouvoir continuer à tirer l'essentiel de leur subsistance de leur milieu de vie malgré les changements climatiques, ont développé diverses stratégies d'adaptation. Ces stratégies assez variées au sein de la communauté paysanne sont aussi bien collectives qu'individuelles avec un fort enracinement dans les savoirs locaux et les progrès de la recherche scientifique en milieu agricole. Ainsi, des stratégies collectives notamment l'association de cultures sont communes aux différentes zones d'étude et interviennent dans l'adaptation des cultivateurs aux variations saisonnières. Selon Willey (1979), l'association de cultures est la culture simultanée de deux (2) espèces ou plus, sur la même surface, pendant une période significative de leur cycle de croissance. Dans les associations de cultures, les espèces associées établissent des interactions négatives (compétition) ou positives (facilitation et complémentarité de niche) pour exploiter les ressources du milieu. Les associations de cultures sont utilisées depuis l'aube de l'agriculture. Mais ces dernières années, elles ont progressivement connu de réels développements à cause des préoccupations des cultivateurs qui cherchent à allier l'efficience des facteurs de production, la préservation de l'environnement et la biodiversité.

L'association des cultures est une des stratégies développées par les cultivateurs de Fresco pour faire face aux effets négatifs des changements climatiques. L'un des avantages de cette méthode est l'exploitation d'une gamme de cultures dont les cultivateurs disposent. Cette diversité, qui inclut des variétés photopériodiques, leur permet de s'adapter à la variabilité des pluies et à la diversité des terrains de culture. De plus, cette méthode permet également de renforcer ou restaurer la capacité de production des terres et réduire ainsi les effets des aléas pluviométriques. Ainsi de façon générale, on note une diversité des systèmes de cultures au sein des exploitations des territoires villageois dans le département de Fresco. Cette diversité fait que même si l'extensif domine en situation de risque, celui-ci peut côtoyer l'intensif. C'est ce que l'on observe entre les systèmes de culture pluviale sur les plateaux et les systèmes de contre-saison pratiqués dans les bas-fonds. Cette diversification des activités rurales au sein d'une exploitation agricole a beaucoup évolué au cours des dernières décennies. Alors qu'elle impliquait autrefois différentes populations à l'échelle préfectorale, désormais, elle s'organise principalement à l'échelle de l'exploitation et des unités villageoises. Depuis longtemps et encore aujourd'hui, ces diversités sont assurées par des ruraux ayant une activité agricole et c'est grâce à cette pluriactivité que beaucoup d'exploitations peuvent surmonter les fluctuations de revenus induites par les aléas climatiques. Nous confirmons ces affirmations par les dires des personnes enquêtées : "J'ai planté beaucoup de bananiers dans mon champ de cacao pour créer un peu d'ombre. Mais surtout 
je l'ai fait pour avoir à manger. Le cacao que j'ai planté est une variété améliorée qu'on appelle cacao dix-huit mois. Si je dois attendre dix-huit mois avant d'avoir une récolte comment moi et ma famille nous allons manger? Donc dans le même champ j'ai aussi planté quelques pieds d'ignames », propos de G.K., un cultivateur à Fresco. « Notre coopérative cultive le vivrier. Sur nos parcelles nous avons planté le manioc pour la fabrication de l'attiéké. Mais à côte du manioc on a aussi planté des bananiers. Les bananes sont pour la consommation et l'attiéké pour la commercialisation », propos de N. D., un membre d'une coopérative agricole du vivrier. "Les pluies ne tombent plus comme par le passé. Donc on essaie de trouver les moyens pour avoir à manger. La méthode que le CNRA nous a montrée c'est de planter différentes cultures sur les mêmes parcelles. Selon les agents du CNRA cette méthode présente beaucoup d'avantages comme l'augmentation et la diversification des récoltes, une meilleure protection du sol qui n'est plus exposé aux rayons solaires et des récoltes disponibles à différentes saisons. J'ai donc planté sur ma parcelle le manioc et le maïs, le piment et les aubergines », propos de F.J., un cultivateur à Fresco. À travers ces propos de nos enquêtés, il ressort que, les associations culturales actuellement pratiquées dans le département de Fresco se justifient tant d'un point de vue agronomique qu'économique ; elles favorisent la sécurisation des productions alimentaires et des revenus, l'amélioration de la productivité du travail grâce à une utilisation optimale du sol et une protection des plantations contre l'érosion grâce à une couverture végétale prolongée.

\section{Discussion et Conclusion}

L'étude a porté sur la résilience aux changements climatiques des cultivateurs dans le département de Fresco. Les résultats révèlent que les cultivateurs résilients du département de Fresco exposés aux changements climatiques, adoptent des cultures à cours cycle et l'association de cultures pour lutter contre les effets néfastes des aléas climatiques. Ces résultats confirment l'étude de Clermont-Dauphin (1995) qui indique que les cultivateurs pour s'adapter aux aléas climatiques adoptent les cultures maraîchères, étant donné que ces cultures sont de cycle court (3 à 4 mois) et n'utilisent pas la totalité des éléments fertilisants. Par ailleurs, selon l'auteur ces cultivateurs associent à ces cultures d'autres cultures de cycle long notamment des cultures vivrières bisannuelles (15 à 18 mois), comme le taro. Ainsi, ces cultivateurs se sont réappropriés ces cultures maraîchères en les introduisant dans des associations de cultures vivrières de plus en plus diversifiées et couvrant le sol sur pratiquement deux années complètes. Salack et al. (2006) abordant dans le même sens soutiennent que les cultivateurs adoptent, de façon délibérée, des pratiques visant à réduire les effets des aléas climatiques. Parmi celles-ci, on peut citer la pratique du semis précoce et 
l'utilisation de variétés à cycle court, qui permettent de faire coïncider au mieux le cycle de la culture avec la saison des pluies, et de réduire ainsi la période de stress hydrique en fin de cycle. Corroborant cette idée Bele et al. (2010) soulignent les opportunités qu'offrent les changements climatiques. En effet, se basant sur l'exemple du Cameroun et de la République Démocratique Congo (RDC), ces auteurs soutiennent que les poches de pluies inhabituelles en saison sèche ont des effets positifs sur la croissance des bananiers-plantains. Ce même constat a été fait sur le taro et sur le manioc. Ainsi, en décidant d'intensifier ces cultures pour réduire leur vulnérabilité aux changements climatiques, les communautés sont conscientes qu'elles procèdent simplement à une expérimentation, compte tenu du degré d'incertitude qui entoure les prévisions sur le changement climatique. C'est dans la même logique qu'elles sont en train de profiter de l'assèchement des marécages pour systématiser la culture du maïs de contre saison. D'autres options telles que 1'apiculture, la domestication du gnetum et les arbres à chenilles, constitueront des filets de sécurité alimentaire en prévision des moments les plus critiques.

De plus, l'étude montre que les cultivateurs résilients du département de Fresco exposés aux changements climatiques, adoptent l'association de cultures pour lutter contre les effets néfastes des aléas climatiques. Brochet (2014) rappelle à cet effet que le développement de cette pratique s'inscrit dans les capacités d'innovation des cultivateurs stimulées par les changements climatiques. Cette approche est corroborée par Yssaad \& Belkhodja (2007), qui attestent que l'association de cultures, en particulier céréale-légumineuse, est une des techniques utilisées par les cultivateurs pour s'adapter aux aléas du climat. Cette pratique est destinée selon ces auteurs à améliorer la texture des sols, leur fertilité, leur rendement et garantir une alimentation humaine. Elle peut également réduire les maladies et les attaques d'insectes. En effet, les légumineuses comme le niebe permettent de fixer l'azote dans le sol, bénéfique à la croissance des céréales telles que le mil ou le sorgho. Les légumineuses et les céréales ont en fait besoin d'éléments nutritifs différents, de phosphore pour les premières et d'azote pour les secondes. La concurrence déjà faible entre elles peut-être encore réduite en décalant les dates de semis des deux (2) espèces. Selon Bambara \& al. (2008), l'association de cultures permet également de diminuer la charge de travail, de limiter l'insuffisance de la main-d'œuvre (les agriculteurs cultivent de plus petites surfaces et économisent de l'énergie pour des travaux comme le désherbage par exemple) et de retenir l'humidité dans le sol. De plus, les légumineuses sont réputées pour leur pouvoir fertilisant (Dupriez, 2007).

En conclusion, il ressort de cette étude que les changements climatiques sont un phénomène récurrent en Côte d'Ivoire en général et en particulier dans le département de Fresco. D'autant plus qu'il touche aujourd'hui un nombre de plus en plus croissant de secteurs d'activités. Cette 
détérioration continue des moyens d'existence a poussé davantage de cultivateurs à rechercher des solutions adaptatives durables pour faire face aux défis des changements climatiques. En recherchant une explication pour savoir comment ces cultivateurs parviennent-ils à faire face aux défis des changements climatiques, nous avons posé comme hypothèse de départ, que les cultivateurs résilients du département de Fresco exposés aux changements climatiques, adoptent des cultures à cours cycle et l'association de cultures. Il ressort de nos investigations en dépit de quelques difficultés rencontrées, que les cultivateurs pratiquent les cultures de rente et vivrières. Par ailleurs, l'étude présente que les changements climatiques ont divers effets sur l'agriculture et les populations. Concernant l'agriculture, ils entrainent des modifications pédologiques, des baisses de rendements agricoles, l'exploitation abusive de la forêt, des modifications de la culture agricole, l'abandon des anciennes plantations et des inondations de plantations. Pour les populations, les changements climatiques entrainent une hausse des prix des denrées agricoles, des inondations villageoises, des modifications de la culture alimentaire, une perte de main-d'œuvre agricole et la migration des populations. Face aux conséquences négatives des changements climatiques, les cultivateurs pour résister et s'adapter à ce phénomène développent un ensemble de pratiques comme avoir deux (2) champs pour cultiver différentes plantes, cultiver les boutures ou les semences, utiliser des semences résistantes ou améliorées, user de produits toxiques et phytosanitaires et la diversification de leurs sources de revenu. Ces éléments permettent de se rendre compte que les stratégies adoptées par les cultivateurs sont des stratégies de survie car elles concernent les revenus et la sécurité alimentaire des cultivateurs. Plusieurs facteurs sont à l'origine de la survenue de cette résilience. Il s'agit entre autres des baisses répétées des rendements agricoles, de l'adoption de cultures à cours cycle et l'association des cultures, et l'utilisation de semences améliorées. Ces pratiques résilientes ont en moyenne moins de 10 ans d'adoption et connaissent une augmentation dans le choix des pratiques agricoles adaptatives des cultivateurs. Leurs impacts sont multiples. Elles favorisent l'adhésion des cultivateurs aux coopératives agricoles, la sécurité alimentaire, des exploitations aux produits variés, des conditions favorables de commercialisation, des performances agricoles et une ouverture au commerce international.

Nous pouvons affirmer à la suite de ces résultats que la résilience aux changements climatiques des cultivateurs de Fresco ramène essentiellement aux actions ou stratégies des communautés locales pour lutter contre les incertitudes du climat et réduire une perception que les conditions ont changé. En outre, on retient que le recours à la tradition, et donc aux anciens rituels, reste encore de mise dans ces communautés. Cette dimension culturelle des stratégies communautaires indiquent que, d'une façon générale, les mesures 
d'adaptation aux changements et à la variabilité climatique développées par les communautés rurales ont un caractère fortement culturel et cultuel. Les populations usent de plusieurs rituels pour la demande et le retour de la pluie. L'adoration des rivières et des terres sacrées et/ou bois sacrés représentent les premiers rituels pratiqués. Le recours aux mesures non rationnelles semble donc prédominer dans les pratiques adaptatives des communautés rurales. Le caractère parfois mystique ou sacré, et surtout la complexité de la relation entre l'homme et le climat expliquent cette tendance à la sacralisation de la nature. L'étude n'a pas abordé de façon spécifique le caractère culturel et cultuel des mesures d'adaptation aux changements climatiques mises en œuvre par les cultivateurs. La connaissance de cette orientation culturelle et cultuelle de ces mesures permettra de percevoir l'influence de la tradition dans la résilience aux changements climatiques des cultivateurs de Fresco. Cet approfondissement du travail est important pour les perspectives d'une recherche future.

\section{References:}

1. Bambara, D., Zoundi, J.S. \& Tiendrébéogo, J-P. (2008). Association céréal/légumineuse et intégration agriculture-élevage en zone soudano-sahélienne. Cahiers agricultures, vol 17, n³, mai-juin, p 297301.

2. Bambara, D.A., Bilgo, E., Hien, D., Masse, A., Thiombiano \& Hien, V. (2013). Perceptions paysannes des changements climatiques et leurs conséquences socio environnementales à Tougou et Donsin, climats sahélien et sahélo soudanien du Burkina Faso, Bulletin de la Recherche Agronomique du Bénin (BRAB), Numéro 74, p 8-16.

3. Bennett. J.W. (2005). The Ecological Transition: Cultural Anthropology and Human Adaptation. New Brunswick (New Jersey): Transaction Publishers, 378 p.

4. Brochet, M. (2014). Des innovations paysannes réussies : associations de cultures maraichères et vivrières ", Field Actions Science Reports [Online], Special Issue 9 | Online since 27 December 2013, connection on $04 \quad$ August $2018 . \quad$ URL : http://journals.openedition.org/factsreports/2793, consulté le 17 mars 2018.

5. Brown, O., A. \& Crawford. (2008). Évaluation des conséquences des changements climatiques sur la sécurité en Afrique de l'Ouest : Étude de cas nationale du Ghana et du Burkina Faso. IIDD, Canada, 74 p; www.iisd.org/pdf/2008/security_implications_west_africa_fr.pdf, consulté le 15 février 2018

6. CEA (2011).Impact, vulnérabilité et adaptation aux changements climatiques en Afrique centrale, 
http://www.uneca.org/ac/Climate\%20change/Impact\%20et\%20vulner abilite.pdf., consulté le 10 mars 2018.

7. Ciccone, A. (1998). L’observation clinique. Paris: Dunod. 117 p.

8. Clark, D. (2006).Climate Change and Social/Cultural Values in the Southwest Yukon: À Resilience Building Perspective, pour le Northern Climate Exchange, Waterloo, 41 p.

9. Clermont -Dauphin, C. (1995). Fertilisation minérale d'une association haricot-maïs en zone tropicale humide. Diagnostic des effets des systèmes de culture. Thèse de docteur de l'INA-PG 297 pages.

10. Convention Cadre des Nations Unies sur les Changements Climatiques (CCNUCC). (2008).10 décembre. http://www.unfccc.de, consulté le 15 février 2018.

11. Dimon, R. (2008).Adaptation aux changements climatiques : perceptions, savoirs locaux et stratégies d'adaptation des producteurs agricoles des communes de Kandi et de Banikoara au Nord du Bénin. Thèse d'ingénieur agronome, FSA-UAC, 132p.

12. Dupriez, H. (2007). Agriculture et exploitations familiales d'Afrique. 3ème édition. 470 pages.

13. Institut National de la Statistique. (2001). Rapport du Recensement National de l'Agriculture, Volet exploitation traditionnelle, $8 \mathrm{p}$.

14. Kanté, A. (2011). Des outils participatifs pour mieux comprendre les liens entre migration et changements climatiques. In Symposium sur les changements climatiques. Panel 3 : Le rôle des savoirs locaux et autochtones dans l'enjeu du changement climatique, AfricAdapt (eds.), $\quad$ p. 3-8 ; adapt.net/media/resources/551/Panel\%203.pdf, consulté le 18 mars 2018.

15. Marema, T. T. \& Crowley. J. (2014). Impact des changements environnementaux sur les migrations humaines, Étude de cas : Sénégal et Côte d'Ivoire, Juin, UNESCO, 294p.

16. Mbalamona, E. A. (2015). Impact des changements climatiques sur l'agriculture et l'environnement dans le bassin du Congo.7ème Conférence Africaine sur la Population Johannesburg Afrique du Sud 30 Novembre - 4 décembre, Editions, Centre d'Etudes et de recherche sur les Analyses et Politiques Economiques (CERAPE), 23p.

17. Ministère de l'Agriculture et du Développement Rural (MINADER). (2006). «Stratégie de Développement du Secteur Rural ». Synthèse du volet agriculture et développement rural, Document de travail, République du Cameroun, p 12-36. 
18. Niang, I., (2009). Le changement climatique et ses impacts : les prévisions au niveau mondial.In : IEPF (eds.). Adaptation au changement climatique ; Liaison Énergie-Francophonie, 85 : 13-19.

19. OCDE (Organisation de Coopération et de Développement Economique). (2009). Climate Change and Agriculture: Impacts, Adaptation, Mitigation and Options for the OECD [COM/TAD/CA/ENV/EPOC (13), 86 p,]; http://olisweb.oecd.org/, consulté le 26 mars 2018

20. Rapport du Cabinet britannique Maplecroft sur la vulnérabilité des pays et des villes au réchauffement climatique, publié en 2015.

21. RGPH (Recensement Général de la Population et de l'Habitat) 2014.

22. SACC (2014). Rapport sur les Stratégie Africaine sur les Changements Climatique.Mai-AMCEN-15-REF-11, 84p.

23. Salack, S., Traoré, B. S., \& Sarr, B. (2006). Synthèse sur la collecte, la mise en forme et le stockage des données climatologiques des pays du CILSS, et Etude d'impacts des changements climatiques sur la production agricole au Sahel, Rapport de stage, Centre Régional AGRHYMET, $95 \mathrm{p}$.

24. UICN (2011). Rapport synthèse des études de capitalisation des connaissances, pratiques, stratégies et technologies locales d'adaptation au changement climatique au Burkina Faso, Mali et Sénégal. Version finale. Projet "Intégration de l'adaptation au changement climatique dans les stratégies de réduction de la pauvreté en Afrique de l'Ouest», $22 \mathrm{p}$.

25. Willey, R.W. (1979). - Intercropping. Its importance and research needs. Part 2. Agronomy and research approaches: Field Crop Abstr., $32(2): 73-85$.

26. Yssaad, H.R. \& Belkhodja, M. (2007). Effet de la bentonite sur l'azote assimilable d'un sol sableux cultivé en céréale et en légumineuse. European Journal of Scientific Research, vol 17, n². p198-206. 